\title{
Invariant Sets for Discrete-Time Constrained Linear Systems Using Sliding Mode Approach
}

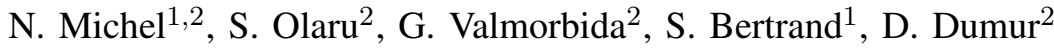

\begin{abstract}
Invariant set theory has been recognized as an important tool for control design of constrained systems subject to disturbances. Indeed, invariant sets as the minimal robustly positively invariant set help verify whether constraints are satisfied in closed-loop. This paper studies a class of discretetime linear systems under additive disturbances. We propose a method to compute the state feedback gain that minimizes the impact of disturbances, related to the mRPI, on the constrained closed-loop dynamics. The method relies on the solution of an optimization problem that is formulated using set-theoretic methods and sliding mode control.
\end{abstract}

\section{INTRODUCTION}

The design of control laws for input and state constrained systems must take into account disturbances and evaluate their impact in closed-loop. A systematic way to assess the influence of disturbance is to compute invariant sets based on a description of the set of disturbance. The invariant set may then be a certificate for robust constraints satisfaction and recursive feasibility [1], and it has been studied in the context of model predictive control [2], robust time-optimal control [3] or design of reference governors [4]. Another important set for constrained systems is the controllable region, which has been studied with different methods [2], [5], [6].

Constrained control in the presence of disturbance faces two main challenges: the characterization of the controllable region and the impact of the disturbance on the convergence towards a nominal equilibrium. These challenges have been addressed in several control design frameworks according to the tools and modelling assumptions: set theoretic methods [5], interval based approaches [6], or Robust Model Predictive Control [2].

The so called minimal Robustly Positively Invariant set (mRPI) is the smallest invariant set for a given set of disturbance and a given feedback control law. It is of particular interest as it corresponds to the limit set of trajectories for any sequence of disturbances. From a constrained controllability point of view, the largest invariant set respecting the constraints, denoted Maximal Robustly Positively Invariant set (MRPI), is also an important notion for any design methodology.

Sliding Mode Control (SMC) has been recognized as one of the effective control strategies for systems with distur-

\footnotetext{
${ }^{1}$ ONERA - The French Aerospace Lab, F-91123 Palaiseau, France. Email: nathan.michel@onera.fr ; sylvain.bertrand@onera.fr

${ }^{2}$ L2S, CentraleSupélec, CNRS, Univ. Paris-Sud, Université ParisSaclay, 3 rue Joliot-Curie, Gif-Sur-Yvette 91192, France. Email: giorgio.valmorbida@12s.centralesupelec.fr ; sorin.olaru@12s.centralesupelec.fr ; didier.dumur@centralesupelec.fr
}

bances [7]. The realization of SMC control for continuoustime systems is performed in two steps. The first step is the design of a sliding surface on which the system exhibits the desired dynamics. The second step is the design of a control law which steers and keeps the system on the sliding surface. For Discrete-time SMC (DSMC), the first step is the same. However, the reaching law is designed to steer and keep the system in a neighborhood of the sliding surface, which we call the quasi-sliding mode band [7]-[9].

This paper presents a method for the synthesis of invariant sets tailored to the constraints of discrete-time linear systems subject to bounded additive disturbance. We use sliding mode to mitigate the impact of the disturbance on the violation of state constraints. The approach proposed here consists in designing a DSMC controller with a Linear Reaching Law that leads to an mRPI as tight as possible in the direction of the constraints. The paper is organized as follows. Section II presents preliminaries and problem formulation. Section III introduces the DSMC framework. Section IV proposes solutions to the problem. Section V gives illustrative examples of the results. Finally, Section VI draws conclusion and discusses perspectives.

Notation: Given two sets $\mathcal{X} \subseteq \mathbb{R}^{n}$ and $\mathcal{Y} \subseteq \mathbb{R}^{n}$, the Minkowski sum and Pontryagin difference are defined as

$$
\begin{aligned}
& \mathcal{X} \oplus \mathcal{Y}=\left\{z \in \mathbb{R}^{n} \mid z=x+y, x \in \mathcal{X}, y \in \mathcal{Y}\right\} \\
& \mathcal{X} \ominus \mathcal{Y}=\left\{x \in \mathbb{R}^{n} \mid\{x\} \oplus \mathcal{Y} \subseteq \mathcal{X}\right\}
\end{aligned}
$$

The $i^{t h}$ power of a matrix $A$ is denoted $A^{i}$, and the $i^{t h}$ row of $A$ is denoted $A_{i}$. $I_{n}$ denotes the identity matrix of dimension $n .0_{m, n}$ denotes the matrix of zeros of dimension $m, n$, or $0_{m}$ if $m=n$. For a matrix $A \in \mathbb{R}^{m, n}$ and a set $\mathcal{X} \subseteq \mathbb{R}^{n}$, define the set $A \mathcal{X}=\left\{y \in \mathbb{R}^{m} \mid y=A x, x \in \mathcal{X}\right\}$. For two real vectors $x$ and $y, x \leq y(x<y)$ denote the elementwise (strict) inequalities between their components. Define the set $\mathbb{R}_{+}^{*}=\{x \in \mathbb{R} \mid x>0\}$. The eigenvalues of matrix $A$ are denoted $\lambda_{i}(A), i=\{1, \ldots, n\}$. The set of Schur matrices of dimension $n$ is defined as $\mathcal{C}_{n}=$ $\left\{A \in \mathbb{R}^{n \times n}|| \lambda_{i}(A) \mid<1, i=\{1, \ldots, n\}\right\}$. Define the set of invertible matrices $\mathcal{G}_{n}=\left\{A \in \mathbb{R}^{n \times n} \mid \operatorname{det}(A) \neq 0\right\}$. For a vector $h \in \mathbb{R}^{n}$, define $\|h\|_{\infty}=\left\{\max \left|h_{i}\right|, i=\{1, \ldots, n\}\right.$. For a matrix $A \in \mathbb{R}^{n \times n}$, define $\|A\|_{\infty}=$ $\left\{\max \left(\sum_{j=1}^{n}\left|A_{i, j}\right|\right), i=\{1, \ldots, n\}, j=\{1, \ldots, n\}\right\}^{\infty}$. 


\section{Problem Statement}

\section{A. System description}

Consider the class of linear discrete-time systems

$$
\begin{aligned}
& x^{+}=A x+B(u+w), \\
& A=\left[\begin{array}{ll}
A_{11} & A_{12} \\
A_{21} & A_{22}
\end{array}\right], B=\left[\begin{array}{c}
0_{n-m, m} \\
I_{m}
\end{array}\right],
\end{aligned}
$$

with $A_{11} \in \mathbb{R}^{(n-m) \times(n-m)}, A_{12} \in \mathbb{R}^{(n-m) \times m}, A_{21} \in$ $\mathbb{R}^{m \times(n-m)}, A_{22} \in \mathbb{R}^{m \times m}, x \in \mathcal{X} \subseteq \mathbb{R}^{n}, u \in \mathbb{R}^{m}$, and $w \in \mathcal{W} \subseteq \mathbb{R}^{m}$, where $x$ is the system state, $u$ is the control input, and $w$ is the exogenous disturbance. Note that any system $x^{+}=\bar{A} x+\bar{B}(u+w)$ with a full rank matrix $\bar{B}$ can be written as (1). The set of state constraints is defined as

$$
\mathcal{X}=\left\{x \in \mathbb{R}^{n} \mid-\underline{g} \leq F x \leq \bar{g}\right\},
$$

where $g \in \mathbb{R}_{+}^{* m}, \bar{g} \in \mathbb{R}_{+}^{* m}$, and $F \in \mathbb{R}^{m \times n}$ can be partitioned as follows

$$
F=\left[\begin{array}{ll}
F_{B^{\perp}} & F_{B}
\end{array}\right],
$$

with $F_{B^{\perp}} \in \mathbb{R}^{m \times(n-m)}$, and $F_{B} \in \mathbb{R}^{m \times m}$. The disturbance set $\mathcal{W}$ is assumed to be polytopic, bounded and to contain the origin in its interior.

Let us introduce a standard definition that will be used to establish criteria for the feedback design.

Definition 1 (Robustly Positively Invariant Set): A set $\mathcal{Z} \subseteq \mathbb{R}^{n}$ is said Robustly Positively Invariant (RPI) for the system (1) with the control law $u=K x$ if $(A+B K) \mathcal{Z} \oplus B \mathcal{W} \subseteq \mathcal{Z}$, i.e.

$$
\forall x \in \mathcal{Z}, \forall w \in \mathcal{W},(A+B K) x+B w \in \mathcal{Z} .
$$

Definition 2 (minimal Robustly Positively Invariant set): The minimal Robustly Positively Invariant (mRPI) set for the system (1) with the control law $u=K x$ is defined as the RPI set contained in any closed RPI set [10]. If $A+B K \in \mathcal{C}_{n}$, the mRPI exists, is compact, contains the origin, and is given by the following infinite Minkowski sum

$$
\mathcal{Z}_{\infty}(K)=\bigoplus_{i=0}^{\infty}(A+B K)^{i} B \mathcal{W} .
$$

Remark 1: The polytopic assumption on $\mathcal{W}$ does not imply $\mathcal{Z}_{\infty}(K)$ to be polytopic. For computational purposes, polytopic RPI outer approximations are sought [11].

Definition 3 (Maximal Robustly Positively Invariant set): The set $\mathcal{O}_{\infty}(K)$ is the maximal RPI (MRPI) for the system (1) with the control law $u=K x$ if it is RPI and if it contains every RPI set under the state constraints (2).

The algorithm proposed in [10] to compute the Maximal Output Admissible Set can be adapted to compute the MRPI

$$
\begin{aligned}
& \mathcal{O}_{0}(K)=\mathcal{X}, \\
& \mathcal{O}_{i+1}(K)=\left\{x \in \mathcal{X} \mid(A+B K) x \in \mathcal{O}_{i}(K) \ominus B W\right\},
\end{aligned}
$$

The algorithm stops if and only if $\mathcal{O}_{\infty}(K)$ is finitely determined. This set defines the region of attraction of the linear feedback gain $K$ under the constraints (2).

\section{B. Problem statement}

The objective of this paper is to propose a methodology to synthesis invariants sets for system (1) that are minimal in the direction of the state constraints (2), that is to compute the linear feedback gain $K$ that minimizes the projection of the mRPI (3) on the constraints $\mathcal{X}$. Let us introduce

$$
h\left(\mathcal{Z}_{\infty}(K)\right)=\left[\begin{array}{lll}
h_{1}\left(\mathcal{Z}_{\infty}(K)\right) & \ldots & h_{m}\left(\mathcal{Z}_{\infty}(K)\right)
\end{array}\right]^{\top},
$$

where

$$
h_{i}\left(\mathcal{Z}_{\infty}(K)\right)=\max _{x \in \mathcal{Z}_{\infty}(K)}\left|F_{i} x\right| .
$$

We note that

$$
\left\|h\left(\mathcal{Z}_{\infty}(K)\right)\right\|_{\infty}=\max _{x \in \mathcal{Z}_{\infty}(K)}\|F x\|_{\infty}=\max _{y \in F \mathcal{Z}_{\infty}(K)}\|y\|_{\infty} .
$$

With the above definitions we can formulate the problem

Problem 1: Given the system (1) and the sets $\mathcal{W}$ and $\mathcal{X}$, find a stabilizing feedback gain $K$ that minimizes $\left\|h\left(\mathcal{Z}_{\infty}(K)\right)\right\|_{\infty}$.

The corresponding optimization problem is the following

$$
\begin{array}{cc}
\underset{K \in \mathbb{R}^{m \times n}}{\operatorname{minimize}} & \max _{y \in F \mathcal{Z}_{\infty}(K)}\|y\|_{\infty} \\
\text { subject to } & A+B K \in \mathcal{C}_{n} .
\end{array}
$$

This optimization problem cannot be solved analytically without an explicit characterization of the set $\mathcal{Z}_{\infty}(K)$ for all stabilizing feedback gains $K$.

In this paper we solve Problem 1 using discrete-time sliding mode control. The framework, as presented in [8], is briefly recalled in the next section. We use set-theoretic methods to show that SMC can be designed to obtain mRPI sets accounting for the constraints $\mathcal{X}$.

The results presented in this paper are obtained under the following assumptions.

Assumption 1: The pair $(A, B)$ is controllable.

Under this assumption, the pair $\left(A_{11},-A_{12}\right)$ is controllable [12].

Assumption 2: $\operatorname{Rank}\left(F_{B}\right)=m$.

\section{Discrete Sliding Mode Control}

To introduce the sliding mode strategy we propose, consider the partition of the state as follows

$$
x=\left[\begin{array}{c}
x_{B^{\perp}} \\
x_{B}
\end{array}\right],
$$

with $x_{B^{\perp}} \in \mathbb{R}^{n-m}, x_{B} \in \mathbb{R}^{m}$. As a first step of the control design procedure, let us consider a matrix $C=$ $\left[\begin{array}{ll}C_{B^{\perp}} & C_{B}\end{array}\right]$, with $C_{B^{\perp}} \in \mathbb{R}^{m \times(n-m)}$, and $C_{B} \in \mathcal{G}_{m}$. This matrix defines the sliding variable $s=C x \in \mathbb{R}^{m}$ and the sliding surface $\mathcal{S}=\left\{x \in \mathbb{R}^{n} \mid C x=0\right\}$.

The following change of coordinates

$$
\xi=Q x=\left[\begin{array}{c}
x_{B^{\perp}} \\
s
\end{array}\right], Q=\left[\begin{array}{cc}
I_{n-m} & 0_{n-m, m} \\
C_{B^{\perp}} & C_{B}
\end{array}\right]
$$

gives an equivalent representation of system (1)

$$
\left.\xi^{+}=\left[\frac{\left[A_{C 1}\right.}{C A Q^{-1}}\right]\right] \xi+\left[\begin{array}{c}
0_{n-m, m} \\
C_{B}
\end{array}\right](u+w),
$$


where $A_{C 1}=A_{11}-A_{12} C_{B}^{-1} C_{B^{\perp}}$. A Linear Reaching Law exploits this structure using the invertibility of $C_{B}$. With

$$
u=-C_{B}^{-1}\left(C A Q^{-1}+\left[\begin{array}{ll}
0_{m, n-m} & A_{C 4}
\end{array}\right]\right) \xi,
$$

in (6), we obtain

$$
\xi^{+}=\underbrace{\left[\begin{array}{cc}
A_{C 1} & A_{12} \\
0_{m, n-m} & A_{C 4}
\end{array}\right]}_{A_{C}} \xi+\left[\begin{array}{c}
0_{n-m, m} \\
C_{B}
\end{array}\right] w .
$$

Matrix $A_{C 4} \in \mathbb{R}^{m \times m}$ is a parameter to be designed. In the original coordinates the control law is given by $u=$ $C_{B}^{-1}\left(-C A+A_{C 4} C\right) x$, therefore defining the linear feedback gain

$$
K=C_{B}^{-1}\left(-C A+A_{C 4} C\right) .
$$

The matrix $A+B K$ is Schur provided

$$
A_{C 1} \in \mathcal{C}_{n-m}, A_{C 4} \in \mathcal{C}_{m}
$$

hold. The definition below relates to the matrices $C$ such that $A_{C 1} \in \mathcal{C}_{n-m}$.

Definition 4: For any pair of matrices $A_{11} \in$ $\mathbb{R}^{(n-m) \times(n-m)}, A_{12} \in \mathbb{R}^{(n-m) \times m}$, the set

$$
\begin{aligned}
& \mathcal{K}\left(A_{11}, A_{12}\right)=\left\{C \in \mathbb{R}^{m \times n} \mid C=\left[\begin{array}{ll}
C_{B^{\perp}} & C_{B}
\end{array}\right],\right. \\
&\left.C_{B} \in \mathcal{G}_{m}, A_{11}-A_{12} C_{B}^{-1} C_{B^{\perp}} \in \mathcal{C}_{n-m}\right\}
\end{aligned}
$$

is called stabilizing set for the pair $\left(A_{11}, A_{12}\right)$.

Remark 2: The change of coordinates (5) and the associated gain (8), leading to $A_{C 4}=0_{m}$, were discussed in [8] and [13]. In these papers the goal was to define stability conditions with regard to the design of the sliding surface and to reject a constant disturbance. This paper instead uses SMC to mitigate the impact the disturbance on the violation of the state constraints.

The mRPI associated to the feedback gain (8) is given by

$$
\begin{aligned}
\mathcal{Z}_{\infty}(K) & =Q^{-1}\left(\bigoplus_{i=0}^{\infty} A_{C}^{i}\left[\begin{array}{c}
0_{m, n-m} \\
C_{B}
\end{array}\right] \mathcal{W}\right) \\
& =Q^{-1}\left(\bigoplus_{i=0}^{\infty}\left[\begin{array}{c}
\sum_{j=0}^{j=i-1} A_{C 1}^{j} A_{12} A_{C 4}^{i-j-1} \\
A_{C 4}^{i}
\end{array}\right] C_{B} \mathcal{W}\right) .
\end{aligned}
$$

Since $C Q^{-1}=\left[\begin{array}{ll}0_{m, n-m} & I_{m}\end{array}\right]$, we have

$$
C \mathcal{Z}_{\infty}(K)=\bigoplus_{i=0}^{\infty} A_{C 4}^{i} C_{B} \mathcal{W}
$$

Remark 3: Note that $C \mathcal{Z}_{\infty}(K)$ depends on the design parameter $A_{C 4}$.

The closed-loop dynamics of the sliding variable $s$ verifies

$$
s^{+}=A_{C 4} s+C_{B} w, w \in \mathcal{W} .
$$

Let $\mathcal{V}_{\infty}\left(A_{C 4}\right) \subseteq \mathbb{R}^{m}$ be the mRPI set for closed-loop system (12), i.e.

$$
\mathcal{V}_{\infty}\left(A_{C 4}\right)=\bigoplus_{i=0}^{\infty} A_{C 4}^{i} C_{B} \mathcal{W}=C \mathcal{Z}_{\infty}(K),
$$

and define the quasi-sliding mode band as the $\mathcal{V}_{\infty}$-vicinity of the sliding surface,

$$
\mathcal{S}_{\mathcal{V}_{\infty}\left(A_{C 4}\right)}=\left\{x \in \mathbb{R}^{n} \mid s=C x \in \mathcal{V}_{\infty}\left(A_{C 4}\right)\right\} .
$$

Proposition 1: The set $\mathcal{S}_{\mathcal{V}_{\infty}\left(A_{C 4}\right)}$ is RPI for the system (1) with the linear feedback gain (8). verifies

Proof: For any $x \in \mathcal{S}_{\mathcal{V}_{\infty}\left(A_{C 4}\right)}$, the sliding variable

$$
s=C x \in \mathcal{V}_{\infty}\left(A_{C 4}\right) .
$$

Given that $\mathcal{V}_{\infty}\left(A_{C 4}\right)$ is RPI for system (12), we have

$$
\forall w \in \mathcal{W}, s^{+}=C x^{+}=A_{C 4} s+C_{B} w \in \mathcal{V}_{\infty}\left(A_{C 4}\right)
$$

Hence, $\forall w \in \mathcal{W}, x^{+} \in \mathcal{S}_{\mathcal{V}_{\infty}\left(A_{C 4}\right)}$.

The results presented above are used in the next section to tackle Problem 1 based on set-theoretic notions and taking into account the structural constraints (9).

\section{Main Results}

Let us introduce the following Lemma

Lemma 1: Consider system (1) and the feedback gain $K \in \mathbb{R}^{m \times n}$ as in (8) with $C \in \mathcal{K}\left(A_{11}, A_{12}\right)$ and $A_{C 4}=0_{m}$. For any feedback gain $L \in \mathbb{R}^{m \times n}$ such that $A+B L \in \mathcal{C}_{n}$, the set inclusion

$$
C \mathcal{Z}_{\infty}(K) \subseteq C \mathcal{Z}_{\infty}(L)
$$

holds.

Proof: Take (11) with $A_{C 4}=0_{m}$ to obtain

$$
C \mathcal{Z}_{\infty}(K)=\bigoplus_{i=0}^{\infty} A_{C 4}^{i} C_{B} \mathcal{W}=C_{B} \mathcal{W}=C B \mathcal{W}
$$

We also have, from (3),

$$
\begin{aligned}
C \mathcal{Z}_{\infty}(L) & =\bigoplus_{i=0}^{\infty} C(A+B L)^{i} B \mathcal{W} \\
& =C B \mathcal{W} \oplus\left(\bigoplus_{i=1}^{\infty} C(A+B L)^{i} B \mathcal{W}\right) \\
& =C \mathcal{Z}_{\infty}(K) \oplus\left(\bigoplus_{i=1}^{\infty} C(A+B L)^{i} B \mathcal{W}\right)
\end{aligned}
$$

Since $0 \in \mathcal{W}, \forall i \in\{1,2, \ldots\}, 0 \in C(A+B L)^{i} B \mathcal{W}$. Hence,

$$
C \mathcal{Z}_{\infty}(K)=C \mathcal{Z}_{\infty}(K) \oplus\{0\} \subseteq C \mathcal{Z}_{\infty}(L)
$$

From this result it is possible to solve Problem 1 under the following assumption on matrix $F$.

Assumption 3: The matrix $F$ satisfies $F \in \mathcal{K}\left(A_{11}, A_{12}\right)$.

Proposition 2: Under Assumption 3,

i) the linear feedback gain $K$ as in (8) with $C=F$ and $A_{C 4}=0_{m}$ solves Problem 1,

ii) if $\mathcal{Z}_{\infty}(K) \subseteq \mathcal{X}$ then the robust asymptotic stability of the set $\mathcal{Z}_{\infty}(K)$ is achieved with a region of attraction $\mathcal{O}_{\infty}(K)=\mathcal{X}$. Moreover, the quasi-sliding mode band $\mathcal{S}_{\mathcal{V}_{\infty}\left(0_{m}\right)}$ is reached in one step.

Proof: i) Let us prove that the linear feedback gain $K$ is a solution of Problem 1 . Since $C=F \in \mathcal{K}\left(A_{11}, A_{12}\right)$, 
then $K$ is a stabilizing feedback gain for (1). Let $L \in \mathbb{R}^{m \times n}$ be a stabilizing feedback gain for (1). According to Lemma 1 with $C=F$,

$$
F \mathcal{Z}_{\infty}(K)=C \mathcal{Z}_{\infty}(K) \subseteq C \mathcal{Z}_{\infty}(L)=F \mathcal{Z}_{\infty}(L)
$$

Hence

$$
\max _{y \in F \mathcal{Z}_{\infty}(K)}\|y\|_{\infty} \leq \max _{y \in F \mathcal{Z}_{\infty}(L)}\|y\|_{\infty} .
$$

This shows that the feedback gain $K$ is a solution of Problem 1.

ii) Since $\mathcal{Z}_{\infty}(K) \subseteq \mathcal{X}$, we have $g \leq F x \leq \bar{g}, \forall x \in$ $\mathcal{Z}_{\infty}(K)$. This relation can be rewritten as

$$
\underline{g} \leq s \leq \bar{g}, \forall s \in F \mathcal{Z}_{\infty}(K) .
$$

Let $x \in \mathcal{X}$. From (12) we have $s^{+}=F_{B} w$. Thus $s^{+} \in$ $F_{B} \mathcal{W}$. Using (11) with $A_{C 4}=0_{m}$, we obtain $F \mathcal{Z}_{\infty}(K)=$ $F_{B} \mathcal{W}$. Thus we have $s^{+} \in \mathcal{Z}_{\infty}(K)$. From (13), we conclude that $g \leq s^{+} \leq \bar{g}$. Therefore, $g \leq F x^{+} \leq \bar{g}$, that is $x^{+} \in \mathcal{X}$. Hence, $\mathcal{X}$ is RPI. From the definition of the MRPI, we have $\mathcal{O}_{\infty}(K)=\mathcal{X}$. Moreover, the set $\mathcal{S}_{\mathcal{V}_{\infty}\left(0_{m}\right)}$ is reached in one step.

We have presented a solution of Problem 1 under the assumption that $F \in \mathcal{K}\left(A_{11}, A_{12}\right)$ using set-theoretic methods.

We now propose a strategy to compute a feedback gain $K$ when Assumption 3 does not hold. The fact that $F \notin$ $\mathcal{K}\left(A_{11}, A_{12}\right)$ prevents us from using the feedback gain $K$ as in (8) with $C=F$ since, in this case, (9) does not hold. The approach proposed here, when Assumption 3 does not hold, is to determine an alternative optimization problem that does not require the computation of the set $\mathcal{Z}_{\infty}(K)$. The underlying strategy is to obtain the closest matrix $C \in \mathcal{K}\left(A_{11}, A_{12}\right)$ to the matrix $F$.

In the following, we impose $K$ as in (8), with $C=$ $\left[\begin{array}{ll}C_{B^{\perp}} & F_{B}\end{array}\right]$. Thus the design parameters are $C_{B^{\perp}}$ and $A_{C 4}$.

Remark 4: Note that using $C=\left[\begin{array}{ll}C_{B^{\perp}} & F_{B}\end{array}\right]$ and $A_{C 4}$ in (8) does not introduce conservatism with respect to the choice $\tilde{C}=\left[\begin{array}{ll}\tilde{C}_{B^{\top}} & \tilde{C}_{B}\end{array}\right], \tilde{A}_{C 4}$ with arbitrary $\tilde{C}_{B}$ since $\tilde{C}_{B^{\top}}=\tilde{C}_{B} F_{B}^{-1} C_{B^{\top}}, \tilde{A}_{C 4}=\tilde{C}_{B} F_{B}^{-1} A_{C 4} F_{B} \tilde{C}_{B^{\top}}$, lead to the same gain $K$.

We have

$$
\begin{aligned}
y & =F x=(F-C) x+C x \\
& =\left(F_{B^{\perp}}-C_{B^{\perp}}\right) x_{B^{\perp}}+C x
\end{aligned}
$$

From (14) and (9), the optimization problem (4) becomes

$$
\begin{array}{cl}
\underset{C_{B} \perp, A_{C 4}}{\operatorname{minimize}} & \max _{x \in \mathcal{Z}_{\infty}(K)}\left\|\left(F_{B^{\perp}}-C_{B^{\perp}}\right) x_{B^{\perp}}+C x\right\|_{\infty} \\
\text { subject to } & C=\left[\begin{array}{ll}
C_{B^{\perp}} & F_{B}
\end{array}\right] \in \mathcal{K}\left(A_{11}, A_{12}\right) \\
& A_{C 4} \in \mathcal{C}_{m} \\
& K=F_{B}^{-1}\left(-C A+A_{C 4} C\right) .
\end{array}
$$

The objective function can be upper-bounded as follows

$$
\begin{array}{r}
\max _{x \in \mathcal{Z}_{\infty}(K)}\left\|\left(F_{B^{\perp}}-C_{B^{\perp}}\right) x_{B^{\perp}}+C x\right\|_{\infty} \\
\leq \max _{x \in \mathcal{Z}_{\infty}(K)}\left\|\left(F_{B^{\perp}}-C_{B^{\perp}}\right) x_{B^{\perp}}\right\|_{\infty} \\
+\max _{x \in \mathcal{Z}_{\infty}(K)}\|C x\|_{\infty} .
\end{array}
$$

The optimization variables $A_{C 4}$ and $C_{B^{\perp}}$ both impact

$$
\max _{x \in \mathcal{Z}_{\infty}(K)}\|C x\|_{\infty}
$$

and

$$
\max _{x \in \mathcal{Z}_{\infty}(K)}\left\|\left(F_{B^{\perp}}-C_{B^{\perp}}\right) x_{B^{\perp}}\right\|_{\infty} .
$$

According to Proposition 2, for a given matrix $C \in$ $\mathcal{K}\left(A_{11}, A_{12}\right)$, (17) is minimized with $A_{C 4}=0_{m}$. Thus, we impose $A_{C 4}=0_{m}$ in the following. Minimizing the upper bound (16) leads to

$$
\begin{array}{cl}
\underset{C_{B^{\perp}}}{\operatorname{minimize}} & \max _{x \in \mathcal{Z}_{\infty}(K)}\left\|\left(F_{B^{\perp}}-C_{B^{\perp}}\right) x_{B^{\perp}}\right\|_{\infty} \\
\text { subject to } & C=\left[\begin{array}{ll}
C_{B^{\perp}} & F_{B}
\end{array}\right] \in \mathcal{K}\left(A_{11}, A_{12}\right) \\
& K=-F_{B}^{-1} C A .
\end{array}
$$

Since the objective function above does not depend on $x_{B}$, using (10) with $A_{C 4}=0_{m}$ leads to the problem

$$
\begin{array}{cl}
\underset{C_{B^{\perp}}}{\operatorname{minimize}} & \max _{B^{\perp} \in \bigoplus_{i=0}^{\infty} A_{C 1}^{i} A_{12} F_{B} \mathcal{W}}\left\|\left(F_{B^{\perp}}-C_{B^{\perp}}\right) x_{B^{\perp}}\right\|_{\infty} \\
\text { subject to } & {\left[C_{B^{\perp}} F_{B}\right] \in \mathcal{K}\left(A_{11}, A_{12}\right)} \\
& A_{C 1}=A_{11}-A_{12} F_{B}^{-1} C_{B^{\perp}}
\end{array}
$$

Since it is not possible to parametrize the set $\bigoplus_{i=0}^{\infty} A_{C 1}^{i} A_{12} F_{B} \mathcal{W}$ in terms of $C_{B^{\perp}}$, we propose to approximate the solution by

a) upper-bounding the eigenvalues of $A_{C 1}$, thus limiting the terms in the set $\bigoplus_{i=0}^{\infty} A_{C 1}^{i} A_{12} F_{B} \mathcal{W}$,

b) minimizing $\left\|F_{B^{\perp}}-C_{B^{\perp}}\right\|_{\infty}$,

relying on the inequality

$$
\left\|\left(F_{B^{\perp}}-C_{B^{\perp}}\right) x_{B^{\perp}}\right\|_{\infty} \leq\left\|F_{B^{\perp}}-C_{B^{\perp}}\right\|_{\infty}\left\|x_{B^{\perp}}\right\|_{\infty} .
$$

Let $\lambda \in] 0,1\left[\right.$, and define $M=\lambda^{-1} A_{C 1}$. If $M \in \mathcal{C}_{n-m}$,

$$
\left|\lambda_{i}\left(A_{C 1}\right)\right|<\lambda, \forall i=\{1, \ldots, n-m\} .
$$

Hence, $C_{B^{\perp}}$ is chosen as the solution of the following optimization problem

$$
\begin{array}{ll}
\underset{C_{B^{\perp}}}{\operatorname{minimize}} & \left\|F_{B^{\perp}}-C_{B^{\perp}}\right\|_{\infty} \\
\text { subject to } & \lambda^{-1}\left(A_{11}-A_{12} F_{B}^{-1} C_{B^{\perp}}\right) \in \mathcal{C}_{n-m} .
\end{array}
$$

The constraint in (18) is characterized with the the SchurCohn criterion (see [14] and [15]), as presented below. The characteristic polynomial of a matrix $M \in \mathbb{R}^{(n-m) \times(n-m)}$ is given by

$$
\begin{aligned}
& \operatorname{det}\left(q I_{n-m}-M\right)= \\
& q^{n-m}+a_{1} q^{n-m-1}+\ldots+a_{n-m-1} q+a_{n-m},
\end{aligned}
$$

where $a_{j}=(-1)^{j} m_{j}(M), \forall j=\{1, \ldots, n-m\}$ and $m_{j}(M)$ is the sum of the $\left(\begin{array}{l}n \\ j\end{array}\right)$ leading minors of order $j$ of $M$. These 
leading minors are polynomial in the elements of $C_{B^{\perp}}$. Let us define the following matrix

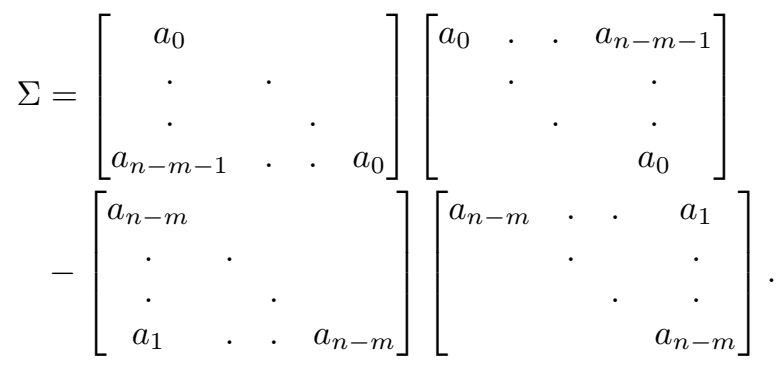

It can be shown that $M \in \mathcal{C}_{n-m}$ if and only if $\Sigma$ is positive definite [14], and that $\Sigma$ is positive definite if and only if its $n-m$ principal leading minors are strictly positive [15]. These principal leading minors are polynomial in the $m_{j}(M), j \in\{1, \ldots, n-m\}$, and thus are polynomial in the coefficients of $C_{B^{\perp}}$. Let $p_{\lambda}\left(C_{B^{\perp}}\right)$ denote the $n-m$ dimensional vector of those $n-m$ polynomials. The matrix verifies $M \in \mathcal{C}_{n-m}$ if and only if $p_{\lambda}\left(C_{B^{\perp}}\right)>0_{m, 1}$.

The optimization problem (18) can then be written as

$$
\begin{array}{ll}
\underset{C_{B^{\perp}}}{\operatorname{minimize}} & \left\|F_{B^{\perp}}-C_{B^{\perp}}\right\|_{\infty} \\
\text { subject to } & p_{\lambda}\left(C_{B^{\perp}}\right)>0_{m, 1} .
\end{array}
$$

We denote its solution $C_{B^{\perp}}^{*}(\lambda)$, and define $C(\lambda)=$ $\left[\begin{array}{ll}C_{B \perp}^{*}(\lambda) & F_{B}\end{array}\right], K(\lambda)$ the linear feedback (8) with $C=$ $C(\lambda)$ and $A_{C 4}=0_{m}, \mathcal{S}_{\mathcal{V}_{\infty}\left(0_{m}\right)}(\lambda)$ the quasi-sliding mode band, and $\mathcal{Z}_{\infty}(K(\lambda))$ the mRPI of the closed-loop system.

Proposition 3: Assume that $\mathcal{Z}_{\infty}(K(\lambda)) \subseteq \mathcal{X}$. The robust asymptotic stability of $\mathcal{Z}_{\infty}(K(\lambda))$ is achieved with an nonempty region of attraction $\mathcal{O}_{\infty}(K(\lambda))$. Finite determination of $\mathcal{O}_{\infty}(K(\lambda))$ is guaranteed. The quasi-sliding mode band $\mathcal{S}_{\mathcal{V}_{\infty}\left(0_{m}\right)}(\lambda)$ is reached in one step.

Proof: By construction, the matrix $A+B K(\lambda)$ is Schur. The proof for the finite determination of $\mathcal{O}_{\infty}(K(\lambda))$ is not reported here, the reader is referred to [10]. By definition of the MRPI, $\mathcal{Z}_{\infty}(K(\lambda)) \subseteq \mathcal{O}_{\infty}(K(\lambda))$, thus the region of attraction is non-empty. The one-step reachability of $\mathcal{S}_{\mathcal{V}_{\infty}\left(0_{m}\right)}(\lambda)$ is deduced from

$$
\forall x \in \mathcal{X}, C x^{+}=F_{B} \mathcal{W} \in \mathcal{V}_{\infty}\left(0_{m}\right)
$$

To verify the assumption $\mathcal{Z}_{\infty}(K(\lambda)) \subseteq \mathcal{X}$, an approach based on outer approximations of the set $\mathcal{Z}_{\infty}(K(\lambda))$ and their support functional [16] is to be privileged. Such an approach avoids the construction of outer approximations of $\mathcal{Z}_{\infty}(K(\lambda))$.

\section{EXAMPLE}

The impact of the design parameter $\lambda$ on $\| h\left(\mathcal{Z}_{\infty}(K(\lambda)) \|_{\infty}\right.$, on the mRPI and on the region of attraction is studied in this section.

Consider the double integrator in regular form

$$
\left[\begin{array}{l}
x_{1} \\
x_{2}
\end{array}\right]^{+}=\left[\begin{array}{ll}
1 & 1 \\
0 & 1
\end{array}\right]\left[\begin{array}{l}
x_{1} \\
x_{2}
\end{array}\right]+\left[\begin{array}{l}
0 \\
1
\end{array}\right](u+w),
$$

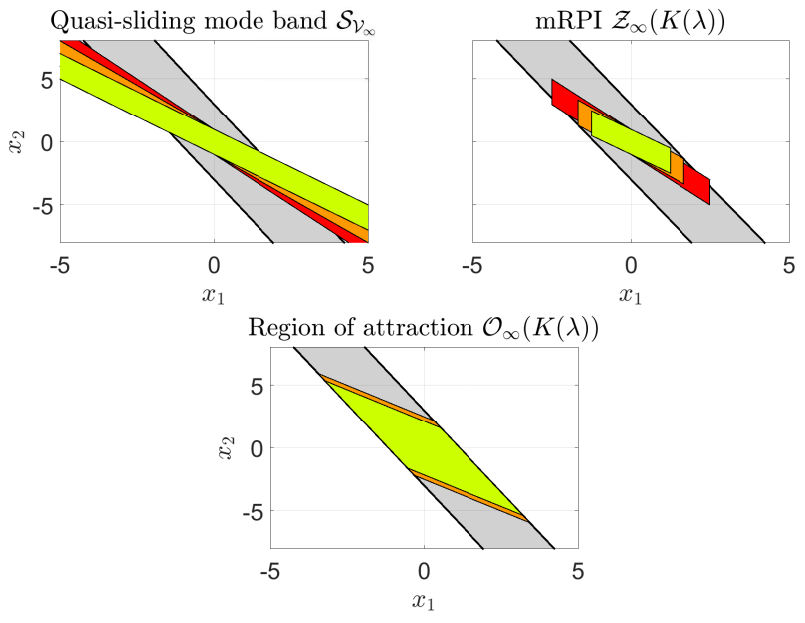

Fig. 1. The set of state constraints $\mathcal{X} 1$ (grey), the quasi-sliding mode bands (top left), the mRPI (top right) and the region of attraction (bottom) for $\lambda=0.2$ (red) $\lambda=0.4$ (orange), and $\lambda=0.6$ (yellow).

hence $A_{11}=A_{12}=1$, with

$$
\mathcal{W}=\{w \in \mathbb{R} \mid-1 \leq w \leq 1\} .
$$

We consider two different state constraints sets as defined in (2),

$$
\begin{aligned}
& \mathcal{X} 1=\{x \mid-\underline{g} \leq F 1 x \leq \bar{g}\}, \mathcal{X} 2=\{x \mid-\underline{g} \leq F 2 x \leq \bar{g}\}, \\
& F 1=\left[\begin{array}{ll}
2.6 & 1
\end{array}\right], F 2=\left[\begin{array}{ll}
-0.1 & 1
\end{array}\right], \bar{g}=\underline{g}=3 .
\end{aligned}
$$

Recall that the proposed method aims at computing the linear feedback gain $K$ that minimizes

$$
\left\|h\left(\mathcal{Z}_{\infty}(K)\right)\right\|_{\infty}=\max _{x \in \mathcal{Z}_{\infty}(K)}\|F x\|_{\infty}
$$

for a given matrix $F$. The matrices $F 1$ and $F 2$ are chosen to violate Assumption $3\left(F \notin \mathcal{K}\left(A_{11}, A_{12}\right)\right.$ ).

The optimization problem (19) is solved for different values of $\lambda$ and successively for the state constraints sets $\mathcal{X} 1$ and $\mathcal{X} 2$. We present the mRPI, the quasi-sliding mode band and the region of attraction, when it exists, in Figure 1 and Figure 2 for $F 1$ and $F 2$ respectively. These figures indicate that the objective function of the original problem (4), $\| h\left(\mathcal{Z}_{\infty}(K(\lambda)) \|_{\infty}\right.$, decreases with $\lambda$, while the region of attraction is enlarged as $\lambda$ increases.

To guarantee robust asymptotic stability of the mRPI it is mandatory to have $\mathcal{Z}_{\infty}(K(\lambda)) \subseteq \mathcal{X}$. Hence, $\lambda$ has to be chosen such that $\| h\left(\mathcal{Z}_{\infty}(K(\lambda)) \|_{\infty} \leq \min (\bar{g}, g)\right.$. Figure 3 shows the relation between $\lambda$ and $\| h\left(\mathcal{Z}_{\infty}(K(\lambda)) \|_{\infty}\right.$, while the red line corresponds to $\| h\left(\mathcal{Z}_{\infty}(K(\lambda)) \|_{\infty}=\min (\bar{g}, \underline{g})\right.$.

These results might indicate that the minimization of $\left\|h\left(\mathcal{Z}_{\infty}(K(\lambda))\right)\right\|_{\infty}$ is obtained by picking $\lambda$ as small as possible. However, this choice can have a negative impact on the size of the region of attraction.

Another indicator of interest for further research on control input constraints handling is

$$
\left\|u\left(\mathcal{Z}_{\infty}(K(\lambda))\right)\right\|_{\infty}=\max _{x \in \mathcal{Z}_{\infty}(K(\lambda))}\|K x\|_{\infty},
$$




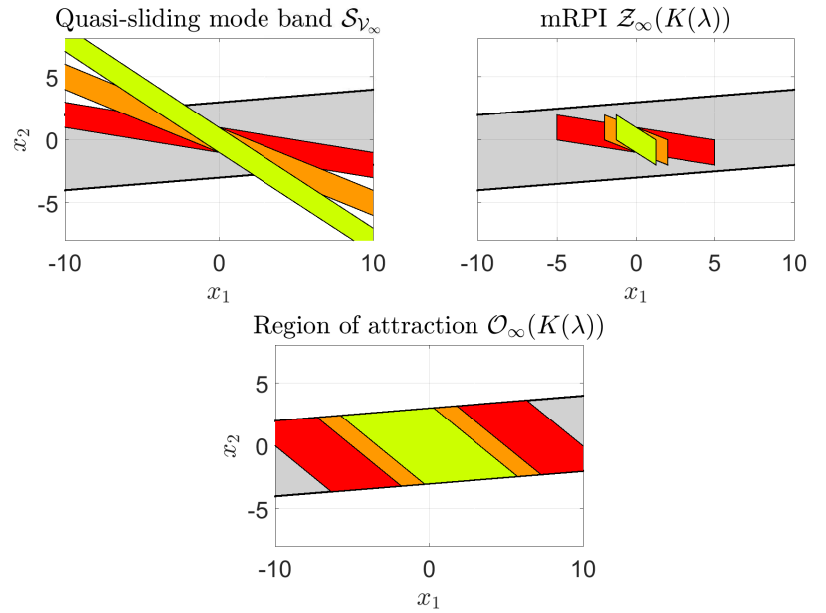

Fig. 2. The set of state constraints $\mathcal{X} 2$ (grey), the quasi-sliding mode bands (top left), the mRPI (top right) and the region of attraction (bottom) for $\lambda=0.2$ (red) $\lambda=0.4$ (orange), and $\lambda=0.6$ (yellow).
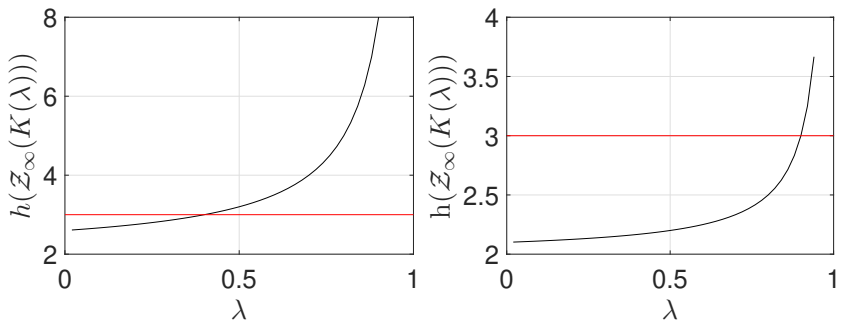

Fig. 3. Relation between $\left\|h\left(\mathcal{Z}_{\infty}(K(\lambda))\right)\right\|_{\infty}$ and $\lambda$ for $\mathcal{X} 1$ (left) and $\mathcal{X} 2$ (right). The red-line corresponds to $h\left(\mathcal{Z}_{\infty}(K(\lambda))\right)=g=\bar{g}=3$.

the maximal control input in the set $\mathcal{Z}_{\infty}(K(\lambda))$. Figure 4 illustrates the relation between the maximal control input value in the mRPI set and $\lambda$ for both state constraints sets $\mathcal{X} i$. It was expected to observe a decrease of $\left\|u\left(\mathcal{Z}_{\infty}(K(\lambda))\right)\right\|_{\infty}$ with the decrease of $\lambda$, but the example shows that such a property can not be generically obtained and should be further investigated.

\section{CONCLUSION}

This paper proposed the design of a state feedback gain for a class of constrained linear systems subject to bounded matched additive disturbance, based on set-theoretic and DSMC frameworks. The proposed strategy was known to
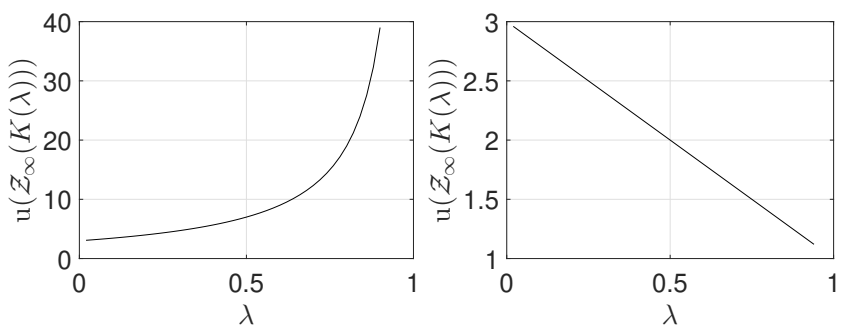

Fig. 4. Relation between $\left\|u\left(\mathcal{Z}_{\infty}(K(\lambda))\right)\right\|$ and $\lambda$ for $\mathcal{X} 1$ (left) and $\mathcal{X}_{2}$ (right). mitigate the impact of the disturbance on the violation of the state constraints. A measure of the impact on the state constraints is proposed and an exact solution is presented where the state constraints matrix belongs in the stabilizing set of a particular pair of matrices in the system characterization. In the general case an alternative optimization-based method is proposed based on Schur matrices characterization. The impact of the scalar design parameter on the minimal and maximal robustly positively invariant sets and on the maximal control input is illustrated. The obtained invariant sets will be used as part of a more complex control strategy, for instance as terminal sets in robust MPC with the goal to extend the region of attraction. Further research includes taking into accounts input constraints.

\section{REFERENCES}

[1] D. Mayne, J. Rawlings, C. Rao, and P. Scokaert, "Constrained model predictive control: Stability and optimality," Automatica, vol. 36, no. 6, pp. $789-814,2000$.

[2] D. Mayne, M. Seron, and S. Rakovic̀, "Robust model predictive control of constrained linear systems with bounded disturbances," Automatica, vol. 41, no. 2, pp. $219-224,2005$.

[3] D. Mayne and W. Schroeder, "Robust time-optimal control of constrained linear systems," Automatica, vol. 33, no. 12, pp. $2103-2118$ 1997.

[4] P. Falcone, F. Borrelli, J. Pekar, and G. Stewart, "A reference governor approach for constrained piecewise affine systems," European Control Conference (ECC), pp. 4223-4228, 2009.

[5] F. Blanchini, "Set invariance in control," Automatica, vol. 35, no. 11, pp. $1747-1767,1999$

[6] L. Jaulin, "Interval constraint propagation with application to boundederror estimation," Automatica, vol. 36, no. 10, pp. 1547 - 1552, 2000.

[7] S. Sarpturk, Y. Istefanopulos, and O. Kaynak, "On the stability of discrete-time sliding mode control systems," IEEE Transactions on Automatic Control, vol. 32, no. 10, pp. 930-932, 1987.

[8] S. K. Spurgeon, "Hyperplane design techniques for discrete-time variable structure control systems," International Journal of Control, vol. 55, no. 2, pp. 445-456, 1992.

[9] W. Gao, Y. Wang, and A. Homaifa, "Discrete-time variable structure control systems," IEEE Transactions on Industrial Electronics, vol. 42, no. 2, pp. 117-122, 1995.

[10] I. Kolmanovsky and E. G. Gilbert, "Theory and computation of disturbance invariant sets for discrete-time linear systems," Mathematical problems in engineering, vol. 4, no. 4, pp. 317-367, 1998.

[11] S. V. Rakovic̀, E. C. Kerrigan, K. I. Kouramas, and D. Q. Mayne, "Invariant approximations of the minimal robust positively invariant set," IEEE Transactions on Automatic Control, vol. 50, no. 3, pp. 406410, 2005.

[12] C. EDWARDS and S. K. SPURGEON, "Sliding mode stabilization of uncertain systems using only output information," International Journal of Control, vol. 62, no. 5, pp. 1129-1144, 1995.

[13] S. Hui and S. H. Zak, "On discrete-time variable structure sliding mode control," Systems \& Control Letters, vol. 38, no. 4, pp. $283-$ 288, 1999.

[14] A. Vieira and T. Kailath, "On another approach to the Schur-Cohn criterion," IEEE Transactions on Circuits and Systems, vol. 24, no. 4, pp. 218-220, 1977.

[15] L. H. Keel, V. A. Oliveira, and S. P. Bhattacharyya, "A new formula for the characteristic equation of a matrix with applications," IEEE Conference on Control Technology and Applications (CCTA), pp. 1631-1636, 2017.

[16] F. Blanchini and S. Miani, Set-theoretic methods in control. Springer, 2008. 